\title{
Multi-stage Stochastic Programming for Demand Response Optimization
}

\author{
Munise Kübra Şahin ${ }^{\mathrm{a}}$, Özlem Çavuş ${ }^{\mathrm{b}}$, Hande Yaman ${ }^{\mathrm{a}}$ \\ ${ }^{a}$ ORSTAT, Faculty of Economics and Business, KU Leuven, 3000 Leuven, Belgium \\ ${ }^{b}$ Department of Industrial Engineering, Bilkent University, 06800 Ankara, Turkey
}

\begin{abstract}
The increase in the energy consumption puts pressure on natural resources and environment and results in a rise in the price of energy. This motivates residents to schedule their energy consumption through demand response mechanism. We propose a multi-stage stochastic programming model to schedule different kinds of electrical appliances under uncertain weather conditions and availability of renewable energy. We incorporate appliances with chargeable and dischargeable batteries to better utilize the renewable energy sources. Our aim is to minimize the electricity cost and the residents' dissatisfaction. We use a scenario groupwise decomposition (group subproblem) approach to compute lower and upper bounds for instances with a large number of scenarios. The results of our computational experiments show that the approach is very effective in finding high quality solutions in small computation times. We provide insights about how optimization and renewable energy combined with batteries for storage result in peak demand reduction, savings in electricity cost and more pleasant schedules for residents with different levels of price sensitivity.
\end{abstract}

Keywords: smart grid, demand response, multi-stage stochastic programming, scenario groupwise decomposition

\section{Introduction}

World energy consumption is expected to grow by $48 \%$ between 2012 and 2040 [1]. The increase in the energy consumption results in a rise in the price of energy by putting pressure on natural resources and environment. Residential energy consumption grows with the population growth accompanied by fewer occupants living

Email addresses: munisekubra.sahin@kuleuven.be (Munise Kübra Şahin), ozlem.cavus@bilkent.edu.tr (Özlem Çavuş), hande.yaman@kuleuven.be (Hande Yaman) 
in larger houses, the change in people's life style and a higher rate of utilization of electrical appliances. Since some of the electrical appliances, like plug-in hybrid vehicles, have a potential to double residential energy consumption, demand side management becomes more crucial. Managing the energy consumption by providing information to residents who can change their consumption patterns is called "demand response" and is one of the demand side management approaches.

Smart grids can play a significant role to provide a sustainable future by integrating residents into the energy saving system through demand response mechanism. With an installation of home energy management system (HEMS), residents can monitor the price information through the smart meters and control their own appliances. By the end of 2016, $47 \%$ of all residential consumers in U.S. have a smart meter in their homes [2]. Since smart meters provide information about the fluctuation of electricity price between low-demand and high-demand hours, with the help of HEMS, residents can shift their demands to the off-peak hours or hours with high renewable energy levels.

The existing studies on scheduling smart home appliances vary in the types of electrical appliances they consider. Based on the energy consumption characteristics, the appliances can be classified under two major types: appliances with continuous or discrete level energy consumption. While the energy consumption amount of the appliances with continuous level energy consumption can vary during the operation, this amount is fixed for the appliances with discrete level energy consumption. In [3], energy consumption scheduling scheme for appliances with continuous level energy consumption is proposed for a distributed generation system. A game theoretic approach is used to formulate the problem where the customers are players whose strategy is to schedule their appliances with the aim of minimizing total energy cost. Later, this study is extended in [4] to consider the peak-to-average ratio (PAR) minimization problem and it is shown that strategies that minimize total energy cost lead to lower PAR values as well. The scheduling of appliances with continuous level energy consumption with the aim of minimizing the summation of disutility and electricity cost of users while keeping total consumption below the generated electricity amount is considered in [5]. Different from these studies, [6] considers the case where the load of appliances with continuous level energy consumption is interruptible. The authors show that if the problem is formulated over a continuous time horizon, although the resulting formulation is non-convex, it has zero duality gap. Therefore, Lagrangian relaxation methods can be used to solve the model. While studies mentioned above consider the deterministic case, uncertainty in the task duration of appliances with continuous level energy consumption is considered in $[7]$. 
The coordination of appliances with discrete level energy consumption is studied in [8] and [9]. While [9] proposes a genetic algorithm, [8] defines a rank-based scheduling heuristic for the fixed price case and force-directed scheduling heuristic for the time-of-use pricing case.

The scheduling of appliances with both continuous and discrete level energy consumption is considered in [10] under price uncertainty. To handle the unknown price parameters, a price prediction capability is added to the proposed model. The differences in the scheduling strategies of appliances both with continuous and discrete level energy consumption for residents with different price sensitivities are investigated in [11].

The energy consumption of appliances that control the temperature of the environment comprises $40 \%$ of total residential energy consumption [12]. This points out the need of scheduling this type of appliances as done by [13], [14], [15], [16], [17] and [18]. However, except [14] and [18], these studies ignore the randomness in weather conditions and model the problem in a deterministic setting. While [15] focuses only on the scheduling of air conditioner/heater and water heater with the aim of minimizing the electricity cost and users' discomfort, [16] incorporates also other types of appliances with continuous level energy consumption in the presence of battery. In addition to appliances that control the temperature of the environment, [13] considers the scheduling of appliances both with continuous and discrete level energy consumption with the aim of minimizing the electricity cost while forcing users' comfort to be greater than a threshold value.

Although one of the main purposes of HEMS is to better utilize the renewable energy, renewable energy is not considered in most of the mentioned studies. It is considered in a deterministic setting in [17], where a single household equipped with appliances with both discrete and continuous level energy consumption is taken into account. Also, the appliances with chargeable and dischargeable batteries are incorporated to the system. Since renewable energy must be consumed at the moment it is produced unless it is stored, using appliances that can store energy helps to increase the use of renewable energy. While [14] proposes a two-stage stochastic programming model considering the uncertainty in renewable energy generation, [7] suggests a three phase algorithm to transform the problem with uncertainties into a set of deterministic problems. In addition to the uncertainty in renewable energy generation, [18] also considers the uncertainty in electric vehicle availability.

Even [7], [14] and [18] address the uncertainty in renewable energy generation, our study is different than these studies in several ways such as the types of electrical appliances considered, the disutility functions and the way to model the uncertainty. As different from [7], [14] and [18], we use multi-stage stochastic programming. Un- 
certainty in renewable energy generation has been considered in multi-stage problems by [19-22]. The scheduling of renewable energy sources and battery usage in a single household is considered in [19]. Later, [20] extends the study [19] for multiple houses where total electricity consumption of other appliances is taken as a random parameter. Multiple houses model is also considered in [22] in the presence of electric vehicle and in [21] where battery storage is also a decision variable. However, these studies focus only on the scheduling of renewable energy sources and battery where the total energy consumption of other appliances is considered as a parameter.

In Table 1, we provide the classification of the studies that are discussed above. In this study, we consider a real-time price based demand response program. For real-time pricing schemes, study [23] shows that checking time-varying prices and changing the energy consumption pattern manually are difficult for most residents due to the lack of time and knowledge. There arises a need for an automated system to better utilize the real-time pricing schemes. For this purpose, we propose a multistage stochastic nonlinear mixed integer programming model that coordinates all types of appliances mentioned above in the presence of weather uncertainty. In particular, our major contributions are the following:

- We propose a novel model to schedule all types of electrical appliances considered in the literature in the presence of renewable energy and batteries.

- We use multi-stage stochastic programming to hedge against the uncertainty in weather conditions, which is critical to model accurately both the energy consumption of the appliances that control the temperature and the availability of the renewable energy.

- Our model is a multi-stage stochastic nonlinear mixed integer programming model. We use a scenario groupwise decomposition (group subproblem) approach to obtain tight lower and upper bounds for instances with large number of scenarios. The results of our computational experiments show that the stochastic demand response optimization problem is very difficult to solve exactly using a general purpose solver. The decomposition approach, on the other hand, gives good quality bounds in reasonable computation times.

- Our computational results show that using the proposed model results in significant improvements in electricity cost and disutility for all types of residents. They also show that even though using battery and renewable energy separately significantly improves the objective function value for all types of residents, residents can make a better use of them when they are combined. 
Table 1: A classification of the studies in the literature

\begin{tabular}{|c|c|c|c|c|c|c|c|c|}
\hline & \multicolumn{2}{|c|}{ Objective } & \multicolumn{5}{|c|}{ Appliance Type } & \multirow{2}{*}{ uncertainty } \\
\hline & $\begin{array}{c}\text { cost } \\
\text { minimization }\end{array}$ & $\begin{array}{l}\text { cost and discomfort } \\
\text { minimization }\end{array}$ & $\begin{array}{r}\mathrm{C} \\
\text { battery }\end{array}$ & $\begin{array}{c}\text { ontinuous Leve } \\
\text { temperature }\end{array}$ & other & $\begin{array}{r}\text { Discr } \\
\text { interruptible }\end{array}$ & $\begin{array}{l}\text { ete Level } \\
\text { uninterruptible }\end{array}$ & \\
\hline$[3]$ & $\checkmark$ & & $x$ & $x$ & $\checkmark$ & $x$ & $x$ & $x$ \\
\hline [4] & $\checkmark$ & & $x$ & $x$ & $\checkmark$ & $x$ & $x$ & $x$ \\
\hline$[5]$ & & $\checkmark$ & $x$ & $x$ & $\checkmark$ & $x$ & $x$ & $x$ \\
\hline$[6]$ & & $\checkmark$ & $x$ & $x$ & $\checkmark$ & $x$ & $x$ & $x$ \\
\hline$[7]$ & & $\checkmark$ & $\checkmark$ & $x$ & $\checkmark$ & $x$ & $x$ & $\checkmark$ \\
\hline$[8]$ & & $\checkmark$ & $x$ & $x$ & $x$ & $x$ & $\checkmark$ & $x$ \\
\hline$[9]$ & & $\checkmark$ & $x$ & $x$ & $x$ & $x$ & $\checkmark$ & $x$ \\
\hline [10] & & $\checkmark$ & $x$ & $x$ & $\checkmark$ & $\checkmark$ & $\checkmark$ & $x$ \\
\hline [11] & & $\checkmark$ & $x$ & $x$ & $\checkmark$ & $x$ & $\checkmark$ & $x$ \\
\hline [13] & $\checkmark$ & & $x$ & $\checkmark$ & $\checkmark$ & $\checkmark$ & $\checkmark$ & $x$ \\
\hline [14] & $\checkmark$ & & $x$ & $\checkmark$ & $\checkmark$ & $\checkmark$ & $\checkmark$ & $\checkmark$ \\
\hline [15] & & $\checkmark$ & $x$ & $\checkmark$ & $x$ & $x$ & $x$ & $x$ \\
\hline [16] & & $\checkmark$ & $\checkmark$ & $\checkmark$ & $\checkmark$ & $x$ & $x$ & $x$ \\
\hline$[17]$ & & $\checkmark$ & $\checkmark$ & $\checkmark$ & $\checkmark$ & $\checkmark$ & $\checkmark$ & $x$ \\
\hline$[18]$ & & $\checkmark$ & $\checkmark$ & $\checkmark$ & $x$ & $\checkmark$ & $\checkmark$ & $\checkmark$ \\
\hline This study & & $\checkmark$ & $\checkmark$ & $\checkmark$ & $\checkmark$ & $\checkmark$ & $\checkmark$ & $\checkmark$ \\
\hline
\end{tabular}

The rest of the paper is organized as follows. In Section 2, we describe the energy consumption characteristics and disutility functions for different appliance types and the electricity pricing scheme. In Section 3, we present our model. In Section 4, we describe the scenario groupwise decomposition approach and present two different ways to construct group subproblems. We present the results of our computational experiments in Section 5. We conclude in Section 6.

\section{Problem Setting}

We consider a smart home equipped with solar panel and electrical appliances that are networked together and controlled by HEMS. While the bidirectional flow of price information is provided by the smart meter, the power can be transmitted only from the energy provider to residents. We consider a discrete-time model with a finite horizon, where scheduling horizon is divided into time slots. The energy consumption scheduling problem aims to achieve a trade-off between minimizing the electricity cost and minimizing the residents' dissatisfaction due to loss of comfort. 


\subsection{Types of Appliances}

Let $\mathcal{A}$ denote the set of appliances networked in this residential unit. An appliance $a$ with discrete level energy consumption only operates in on or off statue and it consumes a fixed energy level of $\bar{e}_{a}$ and $\underline{e}_{a}$ in on and off modes, respectively. Consequently, HEMS decides only when this appliance should be in on mode. On the other hand, for an appliance with continuous level energy consumption, HEMS needs to decide how much energy it consumes in each time period. Each appliance $a$ operates within a user's preferred time interval $T_{a}$ which includes all integers in the range $\left[\underline{t}_{a}, \bar{t}_{a}\right]$.

We classify the appliances with continuous level energy consumption under three categories as follows:

Type 1 : This type of appliances are equipped with chargeable and dischargeable batteries. We denote the set of appliances with battery by $\mathcal{A}_{\mathcal{B}}$.

Type 2: This type of appliances control the temperature of the environment. An example is air conditioner. We denote the set of these appliances by $\mathcal{A}_{\mathcal{T}}$.

Type 3 : This type includes the appliances with continuous level energy consumption that are not of Type 1 and Type 2. Let $\mathcal{A}_{\mathcal{C}}$ denote the set of appliances of Type 3. An example of this type is refrigerator.

We classify the appliances with discrete level energy consumption as follows:

Type 4: This type of appliances can be shut down during operation. This means that their loads are interruptible. An example is a hair dryer. We denote the set of these appliances by $\mathcal{A}_{\mathcal{I}}$.

Type 5 : For this type of appliances, once the operation starts, it should be run to completion. An example is a washing machine. We denote the set of these appliances by $\mathcal{A}_{\mathcal{U}}$.

\subsection{Disutility Functions}

The disutility can have different causes depending on the types of appliances. For an appliance that controls the temperature, the disutility is a result of the discomfort due to low or high inside temperature, whereas for an appliance with discrete consumption, the disutility is a function of earliness of the starting time or the lateness of the finishing time.

The disutility functions for different types of appliances are as follows:

Appliances with discrete level energy consumption (types 4 and 5): For each appliance $a \in \mathcal{A}_{\mathcal{I}} \cup \mathcal{A}_{\mathcal{U}}$, the important decisions are starting and finishing times. The disutility for appliance $a$ if it starts operating at time $\underline{\hat{t}}_{a}$ and finishes at time $\overline{\hat{t}}_{a}$ is given by $\gamma_{a}\left(\underline{\hat{t}}_{a}, \overline{\hat{t}}_{a}\right)$ :

$$
\gamma_{a}\left(\underline{\hat{t}}_{a}, \overline{\hat{t}}_{a}\right)=\underline{\phi}_{D}\left(\left(\underline{t}_{a}^{\text {des }}-\underline{\hat{t}}_{a}\right)_{+}\right)^{2}+\bar{\phi}_{D}\left(\left(\overline{\hat{t}}_{a}-\bar{t}_{a}^{\text {des }}\right)_{+}\right)^{2},
$$


where $\left[\underline{t}_{a}^{\text {des }}, \bar{t}_{a}^{\text {des}}\right]$ represents the most desirable time interval and $\underline{\phi}_{D}$ and $\bar{\phi}_{D}$, respectively, are the weights of disutility for the earliness of the starting time and the lateness of the finishing time. Note that $(x)_{+}:=\max \{x, 0\}$.

Appliances that control temperature (type 2): For appliance $a \in \mathcal{A}_{\mathcal{T}}$, let $h_{a}^{\text {comf }}$ be the most comfortable temperature and $h_{a, t}^{i n}$ be the inside temperature at the location of the appliance in time period $t \in T_{a}$ and $\tau_{a, t}\left(h_{a, t}^{i n}\right)$ be the disutility at time $t$. We use the disutility function used in [16] and [17]:

$$
\tau_{a, t}\left(h_{a, t}^{i n}\right)=\left(h_{a, t}^{i n}-h_{a}^{c o m f}\right)^{2} .
$$

Appliances with battery (type 1): As in [16] and [17], the disutility $\psi_{a, t}\left(r_{a, t}, r_{a, t+1}, b_{a, t}\right)$ for appliance $a \in \mathcal{A}_{\mathcal{B}}$ with battery depends on $r_{a, t}, r_{a, t+1}$ and $b_{a, t}$. Here, $r_{a, t}$ is the power charged to (when $r_{a, t} \geq 0$ ) or discharged from (when $r_{a, t} \leq 0$ ) the appliance in period $t \in T_{a}$ and $b_{a, t}$ is the energy level of the appliance $a$ in period $t$. The disutility is computed as

$$
\psi_{a, t}\left(r_{a, t}, r_{a, t+1}, b_{a, t}\right)=\eta_{1}\left(r_{a, t}\right)^{2}-\eta_{2} r_{a, t} r_{a, t+1}+\eta_{3}\left(\left(\delta \bar{b}_{a}-b_{a, t}\right)_{+}\right)^{2}
$$

where $\eta_{1}, \eta_{2}, \eta_{3}$, and $\delta$ are positive constants and $\bar{b}_{a}$ is the capacity of battery for appliance $a$. The first term penalizes the damaging effect of charging and discharging, the second term penalizes charging-discharging cycles and the third term penalizes deep discharging, which happens when the energy level of the battery drops below $\delta \bar{b}_{a}$. The value of $\delta$ may vary according to the type of battery.

\subsection{Electricity Price}

Since electricity is perishable, the prices set by the electricity retailers fluctuate between low-demand hours and high-demand hours. The most common pricing schemes are real-time pricing (RTP), day-ahead pricing (DAP), time-of-use pricing (TOUP), critical-peak pricing (CPP) and inclining block rates (IBR), (see [10] for further references). In our study, as in [7], [9] and [10], we consider a general hourly pricing function that combines RTP and IBR schemes. It also has a DAP structure as we assume that the future price parameters are known by the residents ahead of time. In our price function, prices vary every hour as in RTP, and beyond a certain threshold for total hourly residential load, the electricity price increases to a higher value as in IBR. Note that, this threshold value is used to decide whether the electricity is charged at a higher price (as used by several power utilities such as California-Pacific Gas and Electric, Southern California Edison, and San Diego Gas and Electric [24]); it does not limit the amount of electricity consumed by a resident. The cost $\rho_{t}\left(u_{t}\right)$ of consuming $u_{t}$ units of electricity in period $t$ is $c_{t}^{l} u_{t}$ if $u_{t} \leq \bar{u}$ and 
$c_{t}^{h} u_{t}$ if $u_{t}>\bar{u}$. Here, $\bar{u}$ is a threshold, $c_{t}^{l}$ is low unit price and $c_{t}^{h}$ is high unit price. In numerical studies, price data of Illinois Power Company and British Columbia (BC) Hydro Company are used (Figure 1).

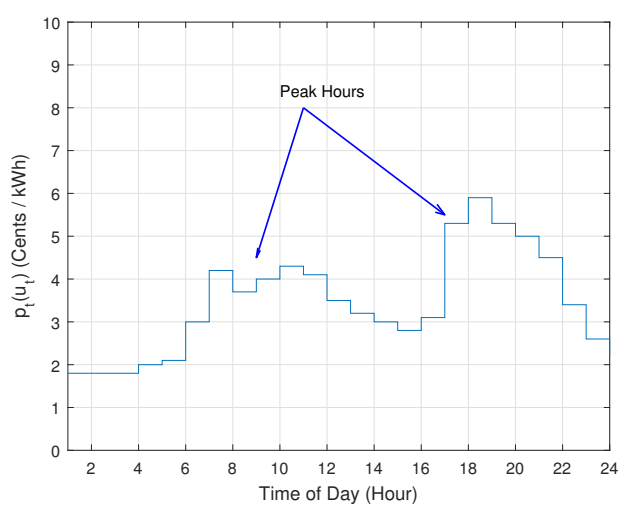

(a) Real-time prices set by Illinois Power Company

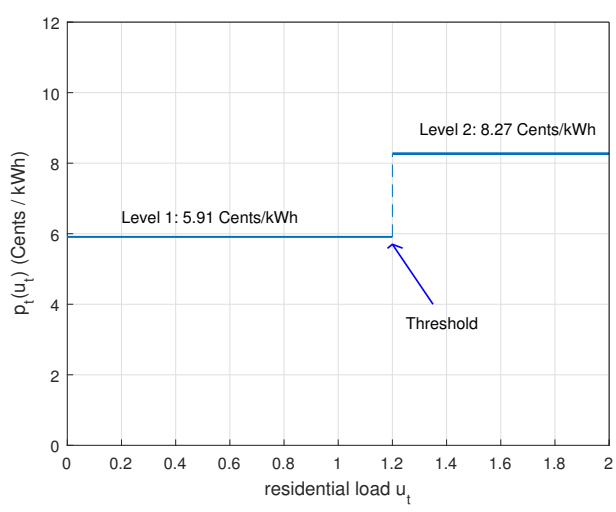

(b) Two-level inclining block rates set by BC Hydro

Figure 1: Examples of two-non flat pricing models. The real time prices are used by Illinois Power Company on December 15 [25]. The inclining block rates are used by BC Hydro Company in December [26]. (This figure is reproduced from [10].)

\section{Multi-stage Stochastic Programming Model}

In this section, we formulate the scheduling problem for smart home appliances as a multi-stage stochastic nonlinear mixed integer program. We assume that the changes in the temperature and in the power of renewable energy are uncertain and this uncertainty is realized gradually at specific time periods called as stages. We also assume that the random temperature and random renewable energy in each stage have discrete distributions with finite number of realizations. Therefore, the uncertainty in the decision process can be represented by a scenario tree. A scenario is defined as a unique path from the root node to a terminal node.

We denote the set of all scenarios by $\mathcal{S}$ and the set of scenarios having the same history as scenario $s$ up to stage $t$ by $\mathcal{S}_{s, t}$. The probabiliy of scenario $s$ is represented by $p(s)$ and the scheduling horizon is represented by $T$. We use the following notation:

\section{Decision Variables}

- $u_{t}^{l}(s)$ : the net energy consumption at low price in period $t \in T$ under scenario $s \in \mathcal{S}$ 
- $u_{t}^{h}(s)$ : the net energy consumption at high price in period $t \in T$ under scenario $s \in \mathcal{S}$

- $b_{a, t}(s)$ : the energy level of appliance $a \in \mathcal{A}_{\mathcal{B}}$ in period $t \in T_{a}$ under scenario $s \in \mathcal{S}$

- $r_{a, t}(s)$ : the power charged/discharged for appliance $a \in \mathcal{A}_{\mathcal{B}}$ in period $t \in T_{a}$ under scenario $s \in \mathcal{S}$

- $e_{a, t}(s)$ : the energy consumption of appliance $a \in \mathcal{A} \backslash \mathcal{A}_{\mathcal{B}}$ in period $t \in T_{a}$ under scenario $s \in \mathcal{S}$

- $e_{a, t}^{+}(s)$ : the energy consumption for heating for appliance $a \in \mathcal{A}_{\mathcal{T}}$ in period $t \in T_{a}$ under scenario $s \in \mathcal{S}$

- $e_{a, t}^{-}(s)$ : the energy consumption for cooling for appliance $a \in \mathcal{A}_{\mathcal{T}}$ in period $t \in T_{a}$ under scenario $s \in \mathcal{S}$

- $x_{a, t}(s): \begin{cases}1, & \text { if appliance } a \in \mathcal{A}_{\mathcal{U}} \cup \mathcal{A}_{\mathcal{I}} \text { starts operation in period } t \in T_{a} \text { under scenario } s \in \mathcal{S} \\ 0, & \text { otherwise }\end{cases}$

- $y_{a, t}(s): \begin{cases}1, & \text { if appliance } a \in \mathcal{A}_{\mathcal{I}} \text { is in on statue in period } t \in T_{a} \text { under scenario } s \in \mathcal{S} \\ 0, & \text { otherwise }\end{cases}$

- $z_{a, t}(s): \begin{cases}1, & \text { if appliance } a \in \mathcal{A}_{\mathcal{I}} \text { completes operation in period } t \in T_{a} \text { under scenario } s \in \mathcal{S} \\ 0, & \text { otherwise }\end{cases}$

- $w_{t}(s): \begin{cases}1, & \text { if energy usage is charged at low price in period } t \in T \text { under scenario } s \in \mathcal{S} \\ 0, & \text { otherwise }\end{cases}$

- $d_{a, t}(s):\left(\delta \bar{b}_{a}-b_{a, t}\right)_{+}$deep discharging amount for appliance $a \in \mathcal{A}_{\mathcal{B}}$ in period $t \in T_{a}$ under scenario $s \in \mathcal{S}$

- $h_{a, t}^{\text {in }}(s)$ : the temperature inside the place of appliance $a \in \mathcal{A}_{\mathcal{T}}$ in period $t \in T_{a}$ under scenario $s \in \mathcal{S}$

\section{Parameters}

- $e_{a}^{\text {tot }} \quad$ : the total energy required for appliance $a \in \mathcal{A}_{\mathcal{C}}$

- $b_{a, 0} \quad$ : the initial energy level of the battery for appliance $a \in \mathcal{A}_{\mathcal{B}}$

- $\bar{r}_{a} \quad$ : the maximum charging amount for appliance $a \in \mathcal{A}_{\mathcal{B}}$ 
- $\underline{r}_{a} \quad$ : the maximum discharging amount for appliance $a \in \mathcal{A}_{\mathcal{B}}$

- $\underline{e}_{a} \quad$ : the minimum energy level required for appliance $a \in \mathcal{A} \backslash \mathcal{A}_{\mathcal{B}}$

- $\bar{e}_{a} \quad$ : the maximum energy level required for appliance $a \in \mathcal{A} \backslash \mathcal{A}_{\mathcal{B}}$

- $\bar{b}_{a} \quad$ : the battery capacity for appliance $a \in \mathcal{A}_{\mathcal{B}}$

- $\underline{h}_{a} \quad$ : the minimum comfortable temperature level for appliance $a \in \mathcal{A}_{\mathcal{T}}$

- $\bar{h}_{a} \quad$ : the maximum comfortable temperature level for appliance $a \in \mathcal{A}_{\mathcal{T}}$

- $h_{a}^{\text {comf }}$ : the most comfortable temperature level for appliance $a \in \mathcal{A}_{\mathcal{T}}$

- $v_{t}(s)$ : the power of renewable energy source in period $\mathrm{t} \in T$ under scenario $s \in \mathcal{S}$

- $n_{a} \quad$ : the total number of time slots required for appliance $a \in \mathcal{A}_{\mathcal{I}} \cup \mathcal{A}_{\mathcal{U}}$ to complete its task

- $h_{a, t}^{\text {out }}(s)$ : the temperature outside the location of appliance $a \in \mathcal{A}_{\mathcal{T}}$ in period $t \in T_{a}$ under scenario $s \in \mathcal{S}$

- $\underline{t}_{a}^{\text {des }} \quad$ : the most desirable starting time for appliance $a \in \mathcal{A}_{\mathcal{I}} \cup \mathcal{A}_{\mathcal{U}}$

- $\bar{t}_{a}^{\text {des }} \quad$ : the most desirable finishing time for appliance $a \in \mathcal{A}_{\mathcal{I}} \cup \mathcal{A}_{\mathcal{U}}$

- $h_{a, 0}^{\text {in }}(s)$ : the initial room temperature for appliance $a \in \mathcal{A}_{\mathcal{T}}$ under scenario $s \in \mathcal{S}$

- $\alpha_{a}, \beta_{a}$ : the thermal characteristics of appliance $a \in \mathcal{A}_{\mathcal{T}}$ and the environment in which appliance operates $\left(\beta_{a}>0\right)$

- $p(s)$ : the probability of scenario $s \in \mathcal{S}$

- $\phi \quad$ : the weight of disutility function

- $\underline{\phi}_{D} \quad$ : the weight of disutility for the earliness of the starting time for appliances in $\mathcal{A}_{\mathcal{I}} \cup \mathcal{A}_{\mathcal{U}}$

- $\bar{\phi}_{D} \quad$ : the weight of disutility for the lateness of the finishing time for appliances in $\mathcal{A}_{\mathcal{I}} \cup \mathcal{A}_{\mathcal{U}}$

- $\phi_{T} \quad$ : the weight of disutility function for appliances in $\mathcal{A}_{\mathcal{T}}$ 
- $\phi_{B} \quad$ : the weight of disutility function for appliances in $\mathcal{A}_{\mathcal{B}}$

An efficient energy consumption schedule can be obtained by solving the following model:

$$
\begin{aligned}
& \min \sum_{s \in \mathcal{S}} p(s)\left(\sum_{t \in T}\left(c_{t}^{l} u_{t}^{l}(s)+c_{t}^{h} u_{t}^{h}(s)\right)+\phi\left(\sum_{a \in \mathcal{A}_{\mathcal{I}} \cup \mathcal{A}_{\mathcal{U}}}\left(\underline{\phi}_{D} \sum_{t=\underline{t}_{a}}^{t_{a}^{\text {des }}}\left(t-\underline{t}_{a}^{\text {des }}\right)^{2} x_{a, t}(s)+\bar{\phi}_{D} \sum_{t=\bar{t}_{a}^{\text {des }}}^{\bar{t}_{a}}\left(t-\bar{t}_{a}^{\text {des }}\right)^{2} z_{a, t}(s)\right)\right.\right. \\
& \left.\left.\quad+\phi_{B} \sum_{a \in \mathcal{A}_{\mathcal{B}}} \sum_{t \in T_{a}}\left(\eta_{1}\left(r_{a, t}(s)\right)^{2}-\eta_{2} r_{a, t}(s) r_{a, t+1}(s)+\eta_{3}\left(d_{a, t}(s)\right)^{2}\right)+\phi_{T} \sum_{a \in \mathcal{A}_{\mathcal{T}}} \sum_{t \in T_{a}}\left(h_{a, t}^{\text {in }}(s)-h_{a}^{\text {comf }}\right)^{2}\right)\right)
\end{aligned}
$$

s.t.

$\underline{e}_{a} \leq e_{a, t}(s) \leq \bar{e}_{a}$,

$\forall a \in \mathcal{A}_{\mathcal{C}}, t \in T_{a}, s \in \mathcal{S}$

$\sum_{t \in T_{a}} e_{a, t}(s)=e_{a}^{t o t}$,

$\underline{e}_{a} \leq e_{a, t}^{+}(s) \leq \bar{e}_{a}$,

$\forall a \in \mathcal{A}_{\mathcal{C}}, s \in \mathcal{S}$

$\forall a \in \mathcal{A}_{\mathcal{T}}, t \in T_{a}, s \in \mathcal{S}$

$\underline{e}_{a} \leq e_{a, t}^{-}(s) \leq \bar{e}_{a}$,

$\forall a \in \mathcal{A}_{\mathcal{T}}, t \in T_{a}, s \in \mathcal{S}$

$e_{a, t}(s)=e_{a, t}^{-}(s)+e_{a, t}^{+}(s)$,

$\forall a \in \mathcal{A}_{\mathcal{T}}, t \in T_{a}, s \in \mathcal{S}$

$h_{a, t}^{i n}(s)=h_{a, t-1}^{i n}(s)+\alpha_{a}\left(h_{a, t}^{\text {out }}(s)-h_{a, t-1}^{i n}(s)\right)+\beta_{a}\left(e_{a, t}^{+}(s)-e_{a, t}^{-}(s)\right), \quad \forall a \in \mathcal{A}_{\mathcal{T}}, t \in T_{a}, s \in \mathcal{S}$

$\underline{h}_{a} \leq h_{a, t}^{i n}(s) \leq \bar{h}_{a}$, $\forall a \in \mathcal{A}_{\mathcal{T}}, t \in T_{a}, s \in \mathcal{S}$

$b_{a, t}(s)=\sum_{u=1}^{t} r_{a, u}(s)+b_{a, 0}$

$\forall a \in \mathcal{A}_{\mathcal{B}}, t \in T_{a}, s \in \mathcal{S}$

$0 \leq b_{a, t}(s) \leq \bar{b}_{a}$,

$\forall a \in \mathcal{A}_{\mathcal{B}}, t \in T_{a}, s \in \mathcal{S}$

$\underline{r}_{a} \leq r_{a, t}(s) \leq \bar{r}_{a}$,

$\forall a \in \mathcal{A}_{\mathcal{B}}, t \in T_{a}, s \in \mathcal{S}$

$d_{a, t}(s) \geq \delta \bar{b}_{a}-b_{a, t}(s)$,

$\forall a \in \mathcal{A}_{\mathcal{B}}, t \in T_{a}, s \in \mathcal{S}$ 


$$
\begin{aligned}
& d_{a, t}(s) \geq 0, \\
& r_{a, \bar{t}_{a}+1}(s)=0, \\
& e_{a, t}(s)=\underline{e}_{a}+\left(\bar{e}_{a}-\underline{e}_{a}\right) y_{a, t}(s) \text {, } \\
& \sum_{t \in T_{a}} y_{a, t}(s)=n_{a}, \\
& \sum_{u=t+1}^{\bar{t}_{a}-n_{a}+1} x_{a, u}(s)+y_{a, t}(s) \leq 1, \\
& \sum_{u=\underline{t}_{a}+n_{a}-1}^{t-1} z_{a, u}(s)+y_{a, t}(s) \leq 1, \\
& \sum_{t=\underline{t}_{a}+n_{a}-1}^{\bar{t}_{a}} z_{a, t}(s)=1, \\
& \sum_{t=\underline{t}_{a}}^{\bar{t}_{a}-n_{a}+1} x_{a, t}(s)=1 \\
& e_{a, t}(s)=\underline{e}_{a}+\left(\bar{e}_{a}-\underline{e}_{a}\right)\left(\sum_{u=\max \left\{\underline{t}_{a}, t-n_{a}+1\right\}}^{t} x_{a, u}(s)\right), \\
& u_{t}^{l}(s)+u_{t}^{h}(s) \geq \sum_{a \in \mathcal{A} \backslash \mathcal{A}_{\mathcal{B}}} e_{a, t}(s)+\sum_{a \in \mathcal{A}_{\mathcal{B}}} r_{a, t}(s)-v_{t}(s), \\
& 0 \leq u_{t}^{l}(s) \leq \bar{u} w_{t}(s), \\
& 0 \leq u_{t}^{h}(s) \leq M\left(1-w_{t}(s)\right) \text {, } \\
& e_{a, t}\left(s^{\prime}\right)=e_{a, t}(s) \text {, } \\
& e_{a, t}^{+}\left(s^{\prime}\right)=e_{a, t}^{+}(s), \\
& e_{a, t}^{-}\left(s^{\prime}\right)=e_{a, t}^{-}(s),
\end{aligned}
$$

$\forall a \in \mathcal{A}_{\mathcal{B}}, t \in T_{a}, s \in \mathcal{S}$

$\forall a \in \mathcal{A}_{\mathcal{B}}, s \in \mathcal{S}(14)$

$\forall a \in \mathcal{A}_{\mathcal{I}}, t \in T_{a}, s \in \mathcal{S}$

$\forall a \in \mathcal{A}_{\mathcal{I}}, s \in \mathcal{S}(16)$

$\forall a \in \mathcal{A}_{\mathcal{I}}, t \in\left[\underline{t}_{a}, \bar{t}_{a}-n_{a}\right], s \in \mathcal{S}$

$\forall a \in \mathcal{A}_{\mathcal{I}}, t \in\left[\underline{t}_{a}+n_{a}, \bar{t}_{a}\right], s \in \mathcal{S}$

$\forall a \in \mathcal{A}_{\mathcal{I}}, s \in \mathcal{S}(19)$

$\forall a \in \mathcal{A}_{\mathcal{I}} \cup \mathcal{A}_{\mathcal{U}}, s \in \mathcal{S}$

$\forall a \in \mathcal{A}_{\mathcal{U}}, t \in T_{a}, s \in \mathcal{S}$

$\forall t \in T, s \in \mathcal{S}$

$\forall t \in T, s \in \mathcal{S}$

$\forall t \in T, s \in \mathcal{S}$

$\forall a \in \mathcal{A} \backslash \mathcal{A}_{\mathcal{B}}, s \in \mathcal{S}, s^{\prime} \in \mathcal{S}_{s, t}, t \in T_{a}$

$\forall a \in \mathcal{A}_{\mathcal{T}}, s \in \mathcal{S}, s^{\prime} \in \mathcal{S}_{s, t}, t \in T_{a}$

$\forall a \in \mathcal{A}_{\mathcal{T}}, s \in \mathcal{S}, s^{\prime} \in \mathcal{S}_{s, t}, t \in T_{a}$ 


$$
\begin{aligned}
& r_{a, t}\left(s^{\prime}\right)=r_{a, t}(s), \\
& x_{a, t}(s) \in\{0,1\}, \\
& y_{a, t}(s) \in\{0,1\}, \\
& z_{a, t}(s) \in\{0,1\}, \\
& w_{t}(s) \in\{0,1\}
\end{aligned}
$$

$$
\begin{aligned}
& \forall a \in \mathcal{A}_{\mathcal{B}}, s \in \mathcal{S}, s^{\prime} \in \mathcal{S}_{s, t}, t \in T_{a} \\
& (28) \\
& \forall a \in \mathcal{A}_{\mathcal{I}} \cup \mathcal{A}_{\mathcal{U}}, t \in T_{a}, s \in \mathcal{S}
\end{aligned}
$$

$\forall a \in \mathcal{A}_{\mathcal{I}}, t \in T_{a}, s \in \mathcal{S}$

$\forall a \in \mathcal{A}_{\mathcal{I}}, t \in T_{a}, s \in \mathcal{S}$

$\forall t \in T, s \in \mathcal{S}$

The objective function (1) is the sum of expected electricity cost and expected dissatisfaction.

For appliances of Type 3, constraints (2) and (3), respectively, ensure that energy consumption is between minimum standby power level and maximum power level and that the total energy requirement is provided within the specified time interval.

Constraints (4) - (8) are given for appliances of Type 2. As above, constraints (4) - (6) ensure that energy consumption is between minimum and maximum levels. Constraints (7) are the balance equations for inside temperature and energy consumption. Constraints (8) ensure that the temperature is in the range that user defines as comfortable.

Constraints (9) - (14) are for the appliances of Type 1. Constraints (9) compute the battery energy level at each period. Constraints (10) ensure that the total charge stored in the battery does not exceed its capacity. Constraints (11) bound the battery charging/discharging amount. Constraints (12) and (13) compute the amount of deep discharging. Constraints (14) ensure that disutility does not occur outside the specified time interval.

Constraints (15) - (20) are for appliances of Type 4. Constraints (15) compute the energy consumption: the energy consumption is equal to the minimum standby power level if the appliance is off and it is equal to the maximum power level if the appliance is on. Constraints (16) ensure that the appliance is on for the number of periods required to fully complete its task. Constraints (17) and (18) allow operation of an appliance only between its starting and finishing times. Constraints (19) and (20) ensure that there is one starting and one finishing time for each appliance.

Constraints (20) and (21) are for appliances of Type 5. One starting time is chosen for each appliance due to constraints (20). Once an appliance starts, it operates consecutively for the required number of periods. Constraints (21) compute the energy consumption amount. 
Constraints (22) - (24) compute the energy consumption amount at low and high prices. Constraints (22) give the relation between the net energy request, energy consumption amount, and renewable energy amount. Constraints (23) and (24) decide whether the energy usage is charged at low price with regard to threshold value $\bar{u}$. The value of $\operatorname{big} M$ is taken to be equal to the sum of the maximum energy level of all appliances that are not of Type 1 (appliances with battery) and the maximum charging amount of appliances with battery.

Constraints (25) - (28) are non-anticipativity constraints to ensure that for any stage $t$, decision variables that have a common history of uncertainty till stage $t$ must have the same value at that stage.

Finally, constraints (29) - (32) are variable restrictions.

\section{Solution Method}

Multi-stage stochastic programming problems are in the framework of extremely challenging problems since their size grows exponentially with the number of stages. Our problem has additional difficulties due to nonlinear objective function and integer variables. Scenario groupwise decomposition approach, which is recently proposed by [27] can be used to obtain bounds for multi-stage stochastic integer problems (see also [28] and [29]). In this approach, the problem is divided into smaller problems called as group subproblems. Group subproblems are defined over a reduced number of scenarios and each of them has the same number of stages as the original problem. The problem is solved for each group subproblem separately rather than being solved for all scenarios simultaneously. Besides easy implementation, since it does not rely on restrictive assumptions such as stagewise independency, complete recourse and convexity, scenario groupwise decomposition is applicable for a wide range of problems including our problem.

\subsection{A Lower Bound}

In this section, we provide a lower bound on the optimal value of our problem using the scenario groupwise decomposition approach of [27]. In this method, a lower bound is obtained by solving the problem separately for subsets of the scenarios called as groups. We define the set of groups as a partition of $\mathcal{S}$. Note that $\mathcal{S}$ denotes the set of all scenarios. Let $\hat{\mathcal{S}}$ be the set of groups and $I_{\hat{\mathcal{S}}}$ be the set of indices of groups in $\hat{\mathcal{S}}$. A subset $\hat{\mathcal{S}}_{i} \subseteq \mathcal{S}$ is called as a group for all $i \in I_{\hat{\mathcal{S}}}$ and the collection of these groups $\hat{\mathcal{S}}=\left\{\hat{\mathcal{S}}_{i}\right\}_{i \in I_{\hat{\mathcal{S}}}}$ is called as a scenario partition if it satisfies $\cup_{i \in I_{\mathcal{S}}} \hat{\mathcal{S}}_{i}=\mathcal{S}$ and $\hat{\mathcal{S}}_{i} \cap \hat{\mathcal{S}}_{j}=\emptyset$ for all $\{i, j\} \subseteq I_{\hat{\mathcal{S}}}$ such that $i \neq j$. Note that groups do not have to be disjoint to obtain valid bounds, as mentioned in [27]. However, for ease of 
representation, we consider disjoint groups. The problem defined over a group, with original probabilities of the scenarios, is called as group subproblem.

After a partition is determined, the probability of each scenario $s \in \mathcal{S}$ is adjusted to achieve a lower bound. The probability of each group $\hat{\mathcal{S}}_{i}$ is the summation of the probabilities of scenarios in that group, that is, $p\left(\hat{\mathcal{S}}_{i}\right)=\sum_{s \in \hat{\mathcal{S}}_{i}} p(s)$ for all $i \in I_{\hat{\mathcal{S}}}$. The adjusted probability of each scenario $s$ is $\hat{p}(s)=p(s) / p\left(\hat{\mathcal{S}}_{i}\right)$ for all $s \in \hat{\mathcal{S}}_{i}$ and $i \in I_{\hat{\mathcal{S}}}$, which is the ratio of the probability of the scenario to the probability of its group. Once the adjusted probabilities are calculated, the weighted summation of the optimal values of the group subproblems provides a lower bound for our minimization problem as proved in [27]. Let $z^{*}(\mathcal{S})$ be the optimal value of our problem with scenario set $\mathcal{S}$ and $\underline{z}(\hat{\mathcal{S}})$ be a lower bound on $z^{*}(\mathcal{S})$ obtained using scenario partition $\hat{\mathcal{S}}$. The following inequality is satisfied by all $\hat{\mathcal{S}}$ selections as proved in Proposition 4.4 of [27]:

$$
\underline{z}(\hat{\mathcal{S}})=\sum_{i \in I_{\hat{\mathcal{S}}}} p\left(\hat{\mathcal{S}}_{i}\right) \cdot z^{*}\left(\hat{\mathcal{S}}_{i}\right) \leq z^{*}(\mathcal{S}) .
$$

The quality of the lower bound depends on the selection and size of groups as demonstrated in [27]. To see the effect of different partition selections, we construct the group subproblems with size $\left|\hat{\mathcal{S}}_{i}\right|=|\mathcal{S}| /\left|I_{\hat{\mathcal{S}}}\right|$ for all $i \in I_{\hat{\mathcal{S}}}$ with following two partition methods:

1) Consecutive: We select $|\mathcal{S}| /\left|I_{\hat{\mathcal{S}}}\right|$ consecutive scenarios using the order that they appear in the scenario tree. This collection constructs the first group subproblem. We repeat this procedure for the rest of the scenarios till all of them are selected.

2) Half-and-half: We select $|\mathcal{S}| /\left|I_{\hat{\mathcal{S}}}\right|$ scenarios half of which is taken from the leaf nodes with the smallest indices and half is taken from the largest indices. This collection constructs the first group subproblem. We repeat this procedure for the rest of the scenarios till all of them are selected.

Since the consecutive method constructs the groups with scenarios having more common nodes, it is expected that consecutive method has an advantage in computation time over the half-and-half method per group. On the other hand, grouping scenarios with less common nodes, as in the half-and-half method, shortens the computation time to compute an upper bound as will be explained in detail in Section 4.2 . 


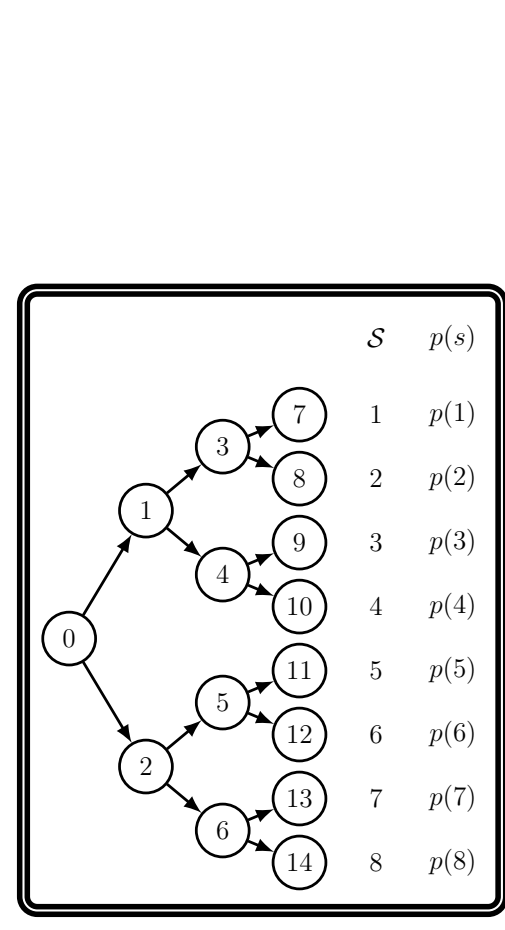

(a) The original problem

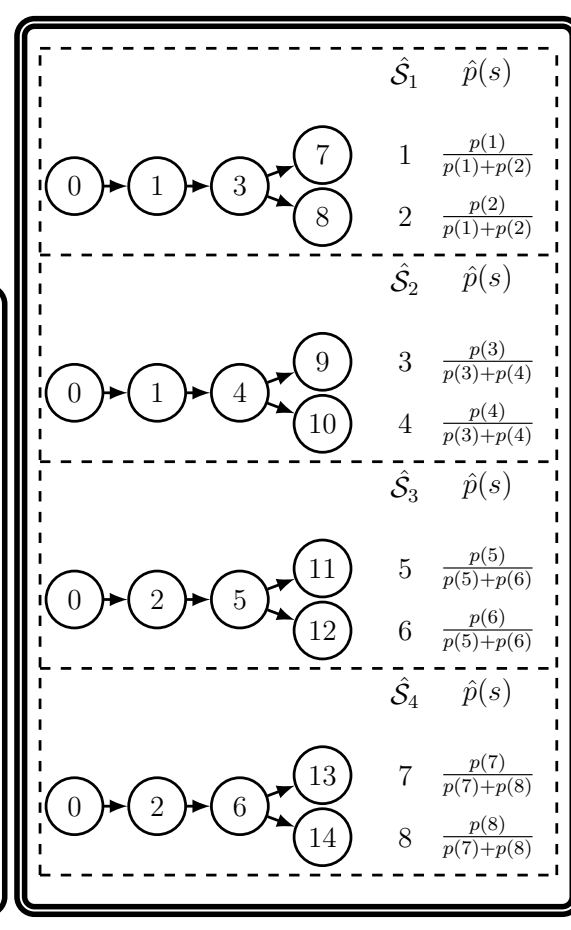

(b) Group subproblems of the consequtive method

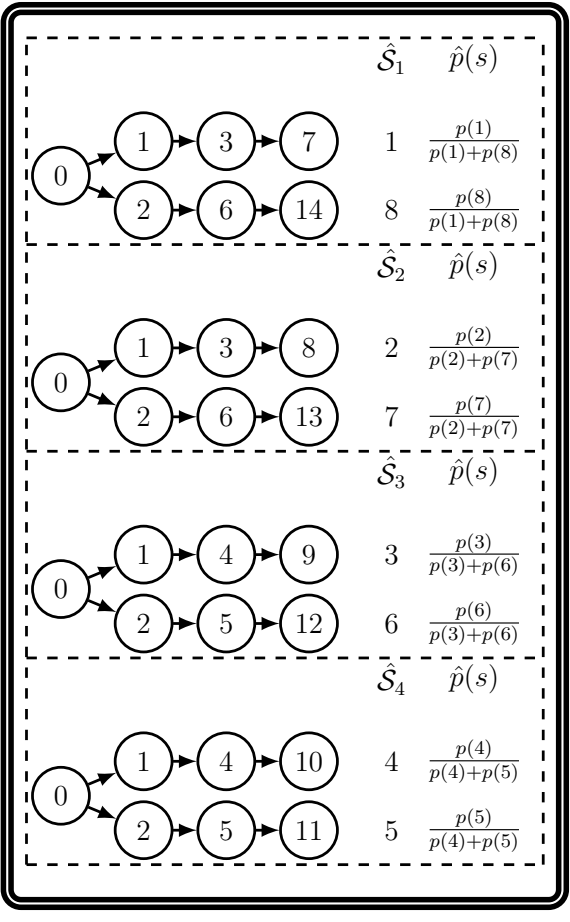

(c) Group subproblems of the half-and-half method

Figure 2: Group subproblems of the consecutive and half-and-half methods for groups of two scenarios for a four stage problem

These partition strategies are explained in Figure 2 over a four stage scenario tree with two branches in each stage. The scenario tree is provided in (a). The construction of groups of size two and the adjusted probabilities of each scenario under this construction are demonstrated in (b) and (c) for the consecutive and half-and-half strategies, respectively.

\subsection{An Upper Bound}

We use an optimal solution of each group subproblem to generate a feasible solution to the original problem as in [27]. Let $x^{*}(S, t)$ be the restriction of an optimal solution of our problem with scenario set $S$ to the first $t$ stages. For a given scenario partition $\hat{\mathcal{S}}$, to obtain an upper bound for our problem, the optimal solution $x^{*}\left(\hat{\mathcal{S}}_{i}, t\right)$ is substituted to the original problem one by one for each group of scenarios $\hat{\mathcal{S}}_{i}, i \in I_{\hat{S}}$. Each resulting problem is called as residual problem and denoted by $\mathcal{P}\left(\hat{\mathcal{S}}_{i}, t\right)$. Then, the residual problems are solved for the remaining decision variables. 
This procedure is explained in Figure 3 for the four stage problem provided in Figure 2. Residual problems obtained after the substitution of $x^{*}\left(\hat{\mathcal{S}}_{1}, 4\right)$ into the original problem are demonstrated in (a) and (b) for consecutive and half-and-half methods, respectively. Note that, residual problems involve only the decision variables and the constraints associated with the non-colored nodes; non-anticipativity constraints regarding the non-colored nodes are kept in the residual problems.

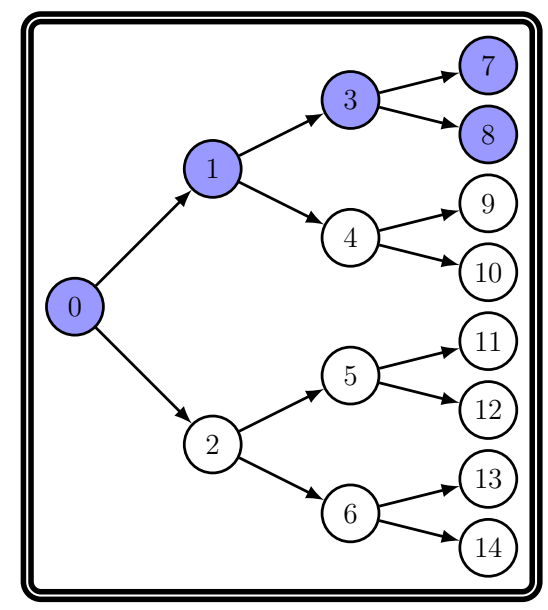

(a) $\mathcal{P}\left(\hat{\mathcal{S}}_{1}, 4\right)$ in consecutive method

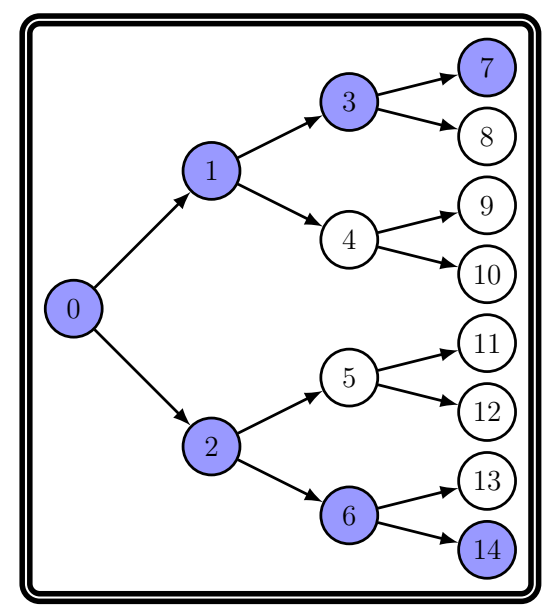

(b) $\mathcal{P}\left(\hat{\mathcal{S}}_{1}, 4\right)$ in half-and-half method

Figure 3: Problems solved to compute an upper bound in different selection strategies

After substitution, if $\mathcal{P}\left(\hat{\mathcal{S}}_{i}, t\right)$ is feasible, its optimal value represented by $z^{*}\left(\hat{\mathcal{S}}_{i}, t\right)$ is a valid upper bound on $z^{*}(\mathcal{S})$. If $\mathcal{P}\left(\hat{\mathcal{S}}_{i}, t\right)$ is infeasible, then we set $z^{*}\left(\hat{\mathcal{S}}_{i}, t\right)$ to $\infty$. Note that, to reduce the possibility of infeasibility, small $t$ values can be used. Additionally, since the number of fixed variables in $\mathcal{P}\left(\hat{\mathcal{S}}_{i}, t\right)$ reduces as $t$ decreases, the resulting bound may get stronger. After solving $\mathcal{P}\left(\hat{\mathcal{S}}_{i}, t\right)$ for all $\hat{\mathcal{S}}_{i}, i \in I_{\hat{S}}$, the minimum of the obtained upper bounds is selected: $z^{*}(\hat{\mathcal{S}}, t)=\min _{i \in I_{\mathcal{S}}} z^{*}\left(\hat{\mathcal{S}}_{i}, t\right)$ and $z^{*}(\hat{\mathcal{S}}, t)$ is the upper bound provided by scenario partition $\hat{\mathcal{S}}$.

The residual problems have less decision variables and constraints compared to the original problem, furthermore, they are decomposable into smaller problems. Therefore, each residual problem may require far less computation time than the original problem. Figure 4 provides the scenario decompositions of the residual problems given in Figure 3. As seen in Figure 4, the consecutive and half-andhalf methods differ with regard to the number and sizes of the problems obtained after decomposition. In the consecutive method, since the scenarios in the group subproblems have more common nodes, the sizes of the problems after decomposition 
are greater. Consequently, we expect that solving the residual problem in the halfand-half method takes less time compared to the consecutive method.

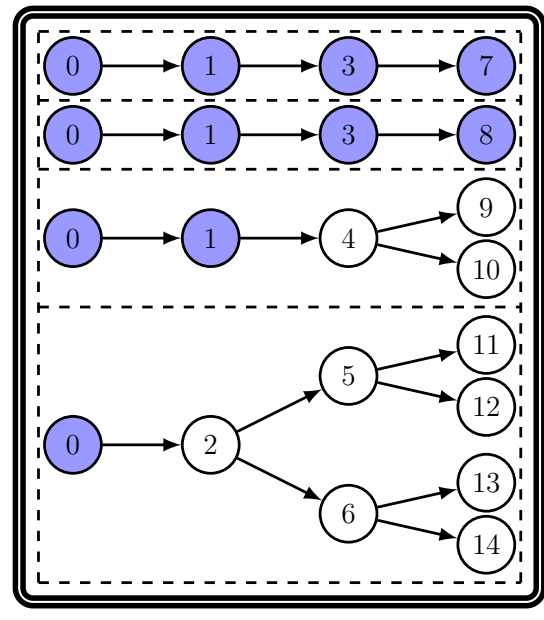

(a) Consecutive method

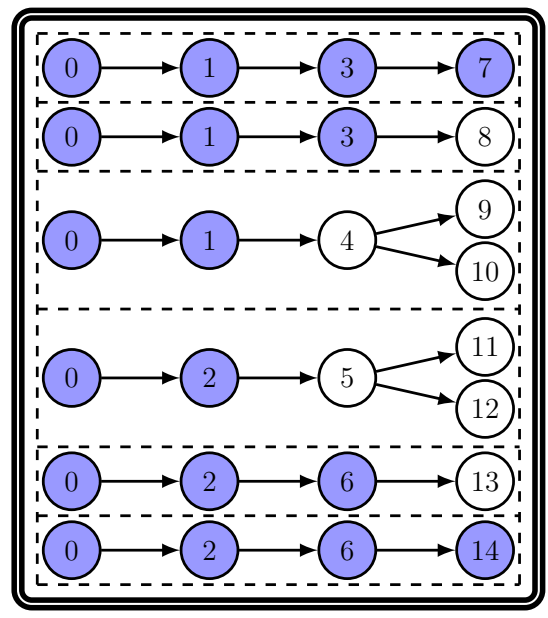

(b) Half-and-half method

Figure 4: Scenario decomposition of $\mathcal{P}\left(\hat{\mathcal{S}}_{1}, 4\right)$ for different selection strategies

\section{Computational Results}

In this section, we present the results of our computational experiments. We first investigate the quality of bounds obtained with the scenario groupwise decomposition. Second, we investigate the gains of demand response optimization for residents with different levels of price sensitivity. Third, we analyze the savings due to the use of renewable energy and batteries.

\subsection{Scenario generation}

We consider a time period of 24 hours and use equal number of periods in each stage. We consider the solar energy as our renewable energy source. The power of renewable energy can be calculated using global horizontal irradiance (GHI) data (total amount of solar irradiance on the horizontal surface of the ground [30]) and the features of the solar panel such as area, tilt angle and the conversion efficiency. To generate the data of temperature and the power of renewable energy randomly, we use the hourly temperature and GHI data obtained from the dataset of National Renewable Energy Laboratory [31]. The correlation between GHI and temperature is calculated as $40 \%$ when considering the daytime data of the year 2005. Despite the positive correlation, high temperature and low amount of solar radiation can be 
observed at the same time because of the solar absorption by clouds, as mentioned in [32] and [33]. We use the real temperature and GHI data for each hour to take into account both the solar absorption and the positive correlation. We provide two data sets generated by the following two methods:

Dataset 1: This data set represents the cases with a good forecast for temperature and GHI. During the data generation process, the data of one month of a specific year are used. Firstly, a random day in the given month is chosen. For each hour of the first stage, corresponding temperature and GHI values of this day are used. When the stage is over, two branches emanate: one branch continues to use the data of this day and for the other branch, the temperature of the last hour of previous stage is compared with the temperature of the same hour of each day in the given month and the data of the day having closest temperature are used. The same procedure is applied for the rest of the stages.

Dataset 2: This data set represents the cases with a relatively bad forecast for temperature and GHI. Different from dataset 1, the data of a given month for different years are used. Data generation process is almost the same as dataset 1: two branches emanate from each node, one branch continues to use the same year data of previous stage and for the other branch, the data of a randomly selected day of a randomly selected year are assigned.

For computational experiments, we consider a smart home equipped with five electrical appliances, each from different types, where HEMS is used to schedule their operations in a day in July.

Our data is available at https://github.com/DemandResponseOptimization/ datasets.

\subsection{Computation times and quality of bounds}

All experiments are carried out on a 64-bit machine with Intel Xeon E5-2630 v2 processor at $2.60 \mathrm{GHz}$ and $96 \mathrm{~GB}$ of RAM using Java and CPLEX 12.6.

First, we analyze the impact of group size and the number of stages for which the variables are fixed on the gap between lower and upper bounds. In Table 2, we report the averages of results for six instances from dataset 1 with six stages (32 scenarios). The first column gives the number of scenarios in each group and the second column gives the number of stages for which we fix the variables to compute an upper bound. We give the computation times and the gaps obtained by the halfand-half and consecutive methods. We set time limit of one hour to compute the lower bound and one hour to compute the upper bound. Since each bound computation requires solution of several problems, we distribute the hour equally for each problem. To compute an upper bound, we set different time limits to each subproblem directly 
proportional to their sizes. If subproblem is not solved to optimality within the time limit, the best bounds are used.

Table 2: Comparion of gaps and solution times for instances with six stages

\begin{tabular}{|c|c|c|c|c|c|}
\hline group size & $\begin{array}{l}\text { number of } \\
\text { stages fixed }\end{array}$ & $\begin{array}{l}\text { half-an } \\
\text { time } \\
(\mathrm{sec})\end{array}$ & $\begin{array}{l}\text { alf method } \\
\text { opt gap } \\
(\%)\end{array}$ & $\begin{array}{l}\text { consecr } \\
\text { time } \\
(\mathrm{sec})\end{array}$ & $\begin{array}{l}\text { ve method } \\
\text { opt gap } \\
(\%)\end{array}$ \\
\hline \multirow{5}{*}{2} & 1 & 2537.47 & 0.57 & 2269.42 & 0.68 \\
\hline & 2 & 63.41 & 0.61 & 1063.85 & 0.63 \\
\hline & 3 & 53.39 & 0.62 & 1057.05 & 0.63 \\
\hline & 4 & 50.97 & 0.62 & 1054.83 & 0.63 \\
\hline & 5 & $\begin{array}{r}49.27 \\
-\end{array}$ & 0.62 & & \\
\hline \multirow{4}{*}{4} & 1 & 2204.91 & 0.49 & 1711.04 & 0.60 \\
\hline & 2 & 122.69 & 0.56 & 842.57 & 0.60 \\
\hline & 3 & 115.6 & 0.58 & 793.80 & 0.60 \\
\hline & 4 & 115.39 & 0.58 & & \\
\hline \multirow[b]{2}{*}{8} & 1 & 2860.53 & 0.63 & 1722.61 & 0.41 \\
\hline & 2 & 1274.68 & 0.71 & 880.56 & 0.45 \\
\hline & 3 & 1270.76 & $\begin{array}{r}0.73 \\
-\end{array}$ & & \\
\hline \multirow{2}{*}{16} & 1 & 4259.95 & 1.92 & 4096.77 & 2.23 \\
\hline & 2 & 3244.75 & 2.01 & & \\
\hline
\end{tabular}

If the half-and-half method is used, the smallest gap is observed when we solve subproblems of four scenarios and fix the variables in the first stage. However, it takes a long time to compute the bounds. The next best case arises again with subproblems of four scenarios but this time we fix the variables in the first two stages and this saves a significant amount of computation time. A better gap is obtained by the consecutive method when the group size is eight and the variables in the first two stages are fixed. However, this again requires a longer computation time compared to the computation time of the half-and-half method with group size of four and two fixed stages. Based on this analysis, we decided to look closely on different instances when we take group subproblems of size four and fix the first two stages. To compute upper bounds, we use the solutions from two randomly selected group subproblems. We report the results in Table 3 for 15 instances. For each instance, we report the lower and upper bounds computed using both selection methods as well as the time required to compute both bounds and the gaps. We also report the optimality gaps given by the solver in the same amount of time. Additionally, in the last column, we report the gaps for the solver with a time limit of one hour. 
Table 3: Comparison of bounds, gaps and solution times for instances with six stages, when groups have four scenarios and the first two stages are fixed in computing upper bounds

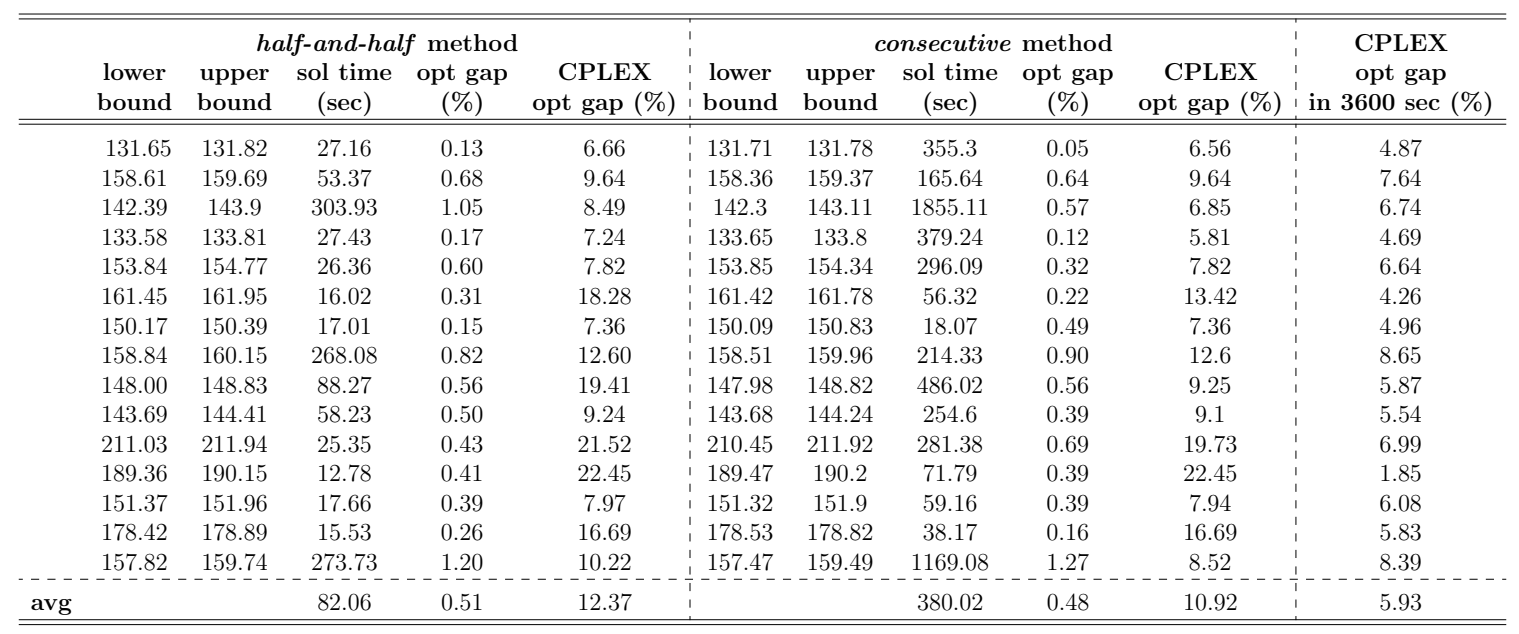

We see that the quality of bounds computed using both selection methods are very similar. However, the half-and-half selection requires a smaller amount of time to compute the bounds. The average gap for the half-and-half method is $0.51 \%$ and the average computation time is 82 seconds. The solver stops with an average gap of $12.37 \%$ in the same amount of time. When given the computation times of the consecutive method, the gap of the solver decreases to $10.92 \%$. After one hour, this gap drops to $5.93 \%$, which is significantly larger than the gap of the scenario groupwise decomposition approaches.

As the half-and-half method gives smaller computation times, we use it to compute bounds for larger instances. In Table 4, we report the results for instances from datasets 1 and 2, respectively, with eight stages (128 scenarios) under different threshold values when each group contains four scenarios, the decisions in the first three stages are fixed and optimal solutions of four randomly selected group subproblems are used in computing upper bounds. We also report the optimality gaps given by the solver in the same amount of time scenario groupwise decomposition approach computes both bounds.

The results show that the decomposition approach is able to compute high quality solutions in reasonable computation times whereas the solver stops with significantly larger gaps in the same amount of time. We also observe that instances from dataset 2 with a smaller threshold are more challenging. 
Table 4: Results for instances with eight stages

\begin{tabular}{|c|c|c|c|c|c|c|}
\hline \multirow[b]{2}{*}{$\bar{u}$} & \multicolumn{3}{|c|}{ dataset 1} & \multicolumn{3}{|c|}{ dataset 2} \\
\hline & $\begin{array}{l}\text { sol time } \\
(\mathrm{sec})\end{array}$ & $\begin{array}{c}\text { opt gap } \\
(\%)\end{array}$ & $\begin{array}{l}\text { CPLEX opt } \\
\text { gap (\%) }\end{array}$ & $\begin{array}{c}\text { sol time } \\
(\mathrm{sec})\end{array}$ & $\begin{array}{c}\text { opt gap } \\
(\%)\end{array}$ & $\begin{array}{l}\text { CPLEX opt } \\
\text { gap (\%) }\end{array}$ \\
\hline \multirow{6}{*}{1.8} & 1962.65 & 0.58 & 9.87 & 2056.24 & 1.80 & 12.53 \\
\hline & 2128.24 & 0.34 & 7.95 & 2491.96 & 1.43 & 7.8 \\
\hline & 2129.39 & 0.72 & 8.96 & 2126.88 & 0.60 & 9.47 \\
\hline & 2210.99 & 0.96 & 10.68 & 2206.33 & 0.85 & 8.08 \\
\hline & 2012.08 & 0.33 & 9.22 & 3594.08 & 0.98 & 7.14 \\
\hline & 1092.17 & 0.51 & 11.56 & 2247.10 & 0.92 & 7.62 \\
\hline avg & 1922.59 & 0.57 & 9.71 & 2453.76 & 1.10 & 8.77 \\
\hline \multirow{6}{*}{2.2} & 618.54 & 0.40 & 6.38 & 807.89 & 1.71 & 8.91 \\
\hline & 53.95 & 0.10 & 1.63 & 721.89 & 0.78 & 16.07 \\
\hline & 51.32 & 0.23 & 1.59 & 1468.1 & 1.11 & 15.02 \\
\hline & 86.14 & 0.59 & 2.34 & 1156.85 & 0.85 & 8.83 \\
\hline & 44.23 & 0.10 & 1.67 & 120.59 & 0.39 & 2.19 \\
\hline & 1163.25 & 0.63 & 10.52 & 1009.09 & 0.89 & 15.13 \\
\hline avg & 336.24 & 0.34 & 4.02 & 880.73 & 0.95 & 11.02 \\
\hline
\end{tabular}

Based on the results of this experiment, we can conclude that the stochastic demand response optimization problem is very difficult to solve exactly using a general purpose solver. Even the instances with six stages are not solved to optimality in an hour.

\subsection{Gains due to demand response optimization}

In our second experiment, we investigate the gains of demand response optimization for residents with different price sensitivity. To this end, we use instances with four stages (eight scenarios), which are all solved to optimality. We set the threshold value to 1.8 for the rest of the experiments. To model different levels of price sensitivity, we vary the disutility weight $\phi$ as $0.1,0.5,1,5$. The value 0.1 models a resident who is very sensitive to price whereas the value 5 models a resident who is not sensitive to price and values his/her comfort.

In Table 5, we report the improvements in the electricity cost and the weighted sum of electricity cost and disutility (our objective function) when we use optimization for four types of residents. For the case without optimization, we schedule the appliances in two ways. First we minimize the discomfort: the continuous appliances use electricity uniformly over the day. The temperature is set to its comfortable value and interruptible and uninterruptible appliances are used in the middle of their desired time intervals. No battery is used, hence the renewable energy is utilized when available. In the case of optimization, we solve our model. We report the average results over ten instances. 
Table 5: The average gains of optimization compared to a schedule that minimizes disutility

\begin{tabular}{l|cccc}
\hline \hline & $\begin{array}{c}\text { very price } \\
\text { sensitive } \\
\text { resident }\end{array}$ & $\begin{array}{c}\text { price } \\
\text { sensitive } \\
\text { resident }\end{array}$ & $\begin{array}{c}\text { less price } \\
\text { sensitive } \\
\text { resident }\end{array}$ & $\begin{array}{c}\text { price } \\
\text { insensitive } \\
\text { resident }\end{array}$ \\
\hline \hline gain in cost (\%) & 41.25 & 26.58 & 22.42 & 17.05 \\
\hdashline \begin{tabular}{l} 
loss in disutility \\
\hdashline
\end{tabular} & 18.65 & 9.49 & 6.72 & 1.73 \\
total gain (\%) & 32.45 & 22.15 & 19.24 & 16.22 \\
\hline \hline
\end{tabular}

We observe that there is significant gain for all types of residents. There is clearly a big difference between the improvements in the electricity cost for residents that are very sensitive and insensitive to price. In the case of price insensitive residents, optimization still reduces the electricity cost by $17 \%$ with a little increase in the disutility.

In the second approach, we schedule the appliances in a greedy way to minimize cost. The continuous appliances operate at maximum energy level during the required number of periods with the lowest electricity price and operate at the remaining energy level at the period with the next lowest electricity price to provide total energy requirement. They operate at minimum energy level for the rest of the day. If the inside temperature is in the allowed range without heating or cooling, the temperature appliance does not operate. Otherwise, the temperature is set to its minimum or maximum comfortable value depending on the outside temperature. Interruptible and uninterruptible appliances are used in time periods that provide the lowest total electricity cost to complete their tasks in their desired interval. No battery is used. We report the average results over ten instances in Table 6.

Table 6: The average gains of optimization compared to a greedy schedule with cost objective

\begin{tabular}{c:cccc}
\hline \hline & $\begin{array}{c}\text { very price } \\
\text { sensitive } \\
\text { resident }\end{array}$ & $\begin{array}{c}\text { price } \\
\text { sensitive } \\
\text { resident }\end{array}$ & $\begin{array}{c}\text { less price } \\
\text { sensitive } \\
\text { resident }\end{array}$ & $\begin{array}{c}\text { price } \\
\text { insensitive } \\
\text { resident }\end{array}$ \\
\hline \hline loss in cost (\%) & 3.08 & 22.38 & 26.64 & 32.89 \\
\hdashline $\begin{array}{c}\text { gain in } \\
\text { disutility(\%) }\end{array}$ & 87.91 & 98.60 & 99.43 & 99.98 \\
\hdashline total gain (\%) & 49.17 & 81.88 & 89.85 & 97.74 \\
\hline \hline
\end{tabular}

We observe that even if there is an increase in the electricity cost, the overall gain is significant for all types of residents as in the previous case. While improvement in disutility is more than $85 \%$ for all types of residents, it is above $99 \%$ for less price sensitive and price insensitive residents. 
These results show that important gains are possible using optimization in demand response for all types of residents.

Table 7: Results on electricity consumption above the threshold, cost and disutility

\begin{tabular}{|c|c|c|c|c|}
\hline & $\begin{array}{l}\text { very price } \\
\text { sensitive } \\
\text { resident }\end{array}$ & $\begin{array}{c}\text { price } \\
\text { sensitive } \\
\text { resident }\end{array}$ & $\begin{array}{c}\text { less price } \\
\text { sensitive } \\
\text { resident }\end{array}$ & $\begin{array}{c}\text { price } \\
\text { insensitive } \\
\text { resident }\end{array}$ \\
\hline electricity & & & & \\
\hline $\begin{array}{l}\text { consumption exceeding } \\
\text { the threshold value }\end{array}$ & 6.81 & 14.63 & 16.80 & 21.10 \\
\hline $\begin{array}{l}\text { total net electricity } \\
\text { consumption }\end{array}$ & 37.87 & 43.79 & 44.77 & 45.67 \\
\hline total cost & 117.71 & 146.77 & 155.61 & 168.76 \\
\hline total disutility & 17.77 & 10.34 & 8.12 & 2.35 \\
\hline
\end{tabular}

Next, we are interested in the tradeoff between the electricity cost and the distutility as well as the amount of electricity consumption exceeding threshold value for different types of residents. In Table 7, we report the average results over ten instances. Since keeping the temperature close to the comfortable value requires more energy than keeping it in the comfortable interval, total electricity consumption increases as price sensitivity of residents decreases. When appliances are used in their most desirable time intervals, the threshold value is exceeded more often and the electricity is charged at the higher price. Consequently, residents who are insensitive to price pay $43.37 \%$ more than the ones who are very sensitive to price while decreasing their disutilities by $86.78 \%$. Almost half of the energy consumption of the price insensitive residents is charged at the higher price whereas the percentage is less than $18 \%$ for residents who are very sensitive to price.

\subsection{Gains due to battery and renewable energy}

In our third experiment, we investigate the impact of battery and renewable energy on the electricity costs and disutilities of residents.

We present average results over ten instances in Table 8. Without the renewable energy, the use of batteries already results in significant improvements in the objective function for all types of residents. The gain due to renewable energy is similar for all resident types and it is a remarkable amount. But even further improvements are possible when the renewable energy is combined with batteries.

To see the impact of the renewable energy and the battery on electricity consumption during a day, we plot the consumption due to different types of appliances for an instance over a scenario in Figure 5. Low and high price values and the temperature and available renewable energy over a day are depicted in (a) and (b), 
Table 8: Results of gains due to battery and renewable energy

\begin{tabular}{c:cccc}
\hline \hline & $\begin{array}{c}\text { very price } \\
\text { sensitive } \\
\text { resident }\end{array}$ & $\begin{array}{c}\text { price } \\
\text { sensitive } \\
\text { resident }\end{array}$ & $\begin{array}{c}\text { less price } \\
\text { sensitive } \\
\text { resident }\end{array}$ & $\begin{array}{c}\text { price } \\
\text { insensitive } \\
\text { resident }\end{array}$ \\
\hline \hline gain due to battery (\%) & 3.16 & 3.48 & 3.67 & 4.21 \\
\hdashline gain due to renewable & 25.31 & 25.10 & 24.82 & 24.84 \\
energy (\%) & 27.11 & 28.34 & 28.66 & 28.74 \\
\hline $\begin{array}{c}\text { gain due to battery and } \\
\text { renewable energy (\%) }\end{array}$ & & & & \\
\hline \hline
\end{tabular}

respectively. From (c) and (d), it can be deduced that using a battery decreases the electricity consumption in time periods with higher prices (between 5 and 10 P.M.). In (c), battery stores electricity when the price is low. While the battery feeds the system when interruptible appliance operates, it stores electricity when it does not operate or when the amount of renewable energy is high. This flexibility enables the appliances to work during their desirable time intervals while keeping total consumption under the threshold value. When the battery is not used, in (d), although the uninterruptible appliance moves away from its desirable time interval, total consumption exceeds the threshold value in a time period with a relatively higher price. The comparison of (c) and (e) shows that when renewable energy is not available, consumption shifts to hours with cheaper price. In addition to the increase in the number of time periods in which electricity is charged at the higher price, the amount charged at this price also increases without renewable energy. Figures (e) and (f) show that the battery provides savings even when renewable energy is not available. While the periods in which the threshold is exceeded are generally those with lower electricity price in (e), when the battery is removed, we encounter also periods with high consumption at relatively higher prices in (f).

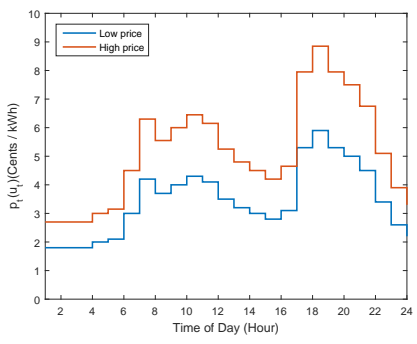

(a) Low and high price values

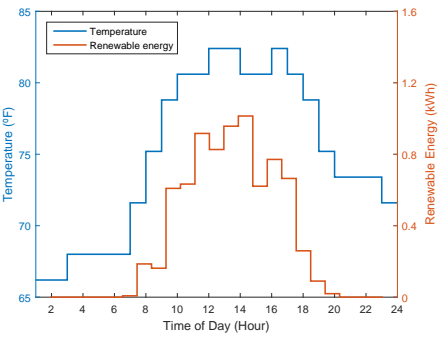

(b) Temperature and renewable energy

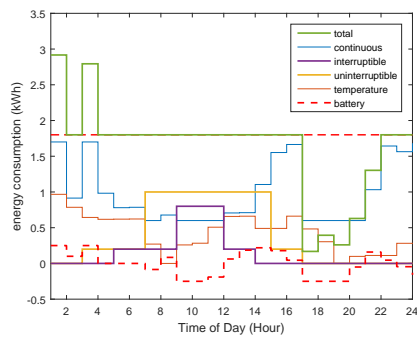

(c) Energy consumption 


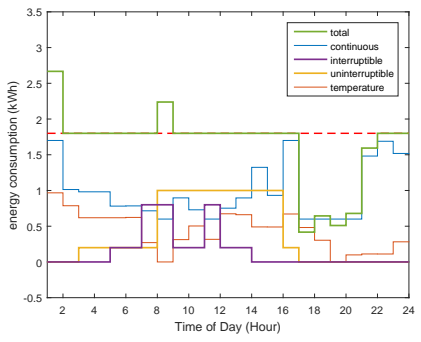

(d) Energy consumption without battery

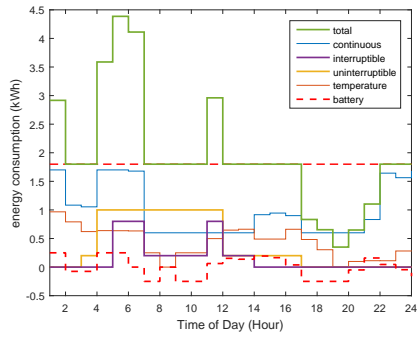

(e) Energy consumption without renewable energy

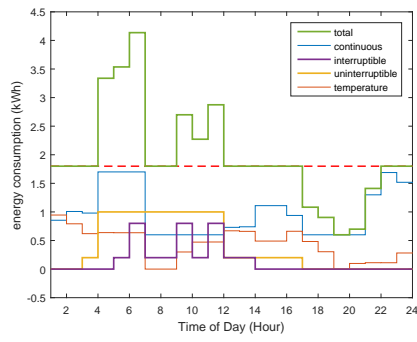

(f) Energy consumption without battery and renewable energy

Figure 5: Effect of battery and renewable energy on the daily consumption

\subsection{Value of Information and Stochastic Solution}

In our final experiment, we compare the performances of stochastic and deterministic solutions via expected value of perfect information $(E V P I)$ and value of stochastic solution $(V S S)$. To calculate $V S S$, we solve the problem in a rolling horizon updating the revealed information in each stage as in [34]. At the first step, we solve a deterministic problem where all uncertain parameters are replaced with their expected values. In the next step, we are interested in the expected cost that the decision maker will incur if $\mathrm{s} /$ he implements the optimal first stage solution of the previous step without considering the uncertainty of future stages. Since the uncertainty related to the second stage is revelaed in the second step, all the uncertain parameters other than the ones corresponding to the second stage are set to their expected values. Then the values of the first stage decision variables are fixed to the optimal first stage solution of the previous step and the resulting problem is solved. This procedure is repeated until the last stage and the optimal value of the problem solved at the last stage (step) is called as $E E V_{\text {rolling. }}$. We report the results in Table 9 for six instances. For each instance, we report the optimal value of stochastic problem $\left(z^{S P}\right)$, wait-and-see value $\left(z^{W S}\right), E V P I$ and $V S S$. Here, the $V S S$ is the difference between $E E V_{\text {rolling }}$ and $z^{S P}$. We also report the results of scenario groupwise decomposition method when each group contains two scenarios and the decisions in the first stage are fixed.

It can be seen that the decision maker may incur on the average $6.12 \%$ increase in the objective function value by using the rolling horizon approach instead of solving the stochastic problem exactly. Therefore, solving the stochastic problem is valuable for the decision maker. Furthermore, the optimality gap of our solution method is $0.01 \%$ on the average. Therefore, even our method does not guarantee to find an exact solution, it provides a solution much better than the one given by the rolling 
Table 9: EVPI and VSS for instances with four stages

\begin{tabular}{|c|c|c|c|c|c|c|c|c|c|c|}
\hline \multirow[b]{2}{*}{$z^{S P}$} & \multirow[b]{2}{*}{$z^{W S}$} & \multirow[b]{2}{*}{$E V P I$} & \multirow[b]{2}{*}{$E V P I(\%)$} & \multirow[b]{2}{*}{$E E V_{\text {rolling }}$} & \multirow[b]{2}{*}{$V S S$} & \multirow[b]{2}{*}{$V S S(\%)$} & \multicolumn{4}{|c|}{ scenario groupwise decomposition method } \\
\hline & & & & & & & $\begin{array}{l}\text { lower } \\
\text { bound }\end{array}$ & $\begin{array}{l}\text { upper } \\
\text { bound }\end{array}$ & $\begin{array}{l}\text { gap between } \\
\text { lower and upper } \\
\text { bound }(\%)\end{array}$ & opt gap (\%) \\
\hline 137.97 & 137.84 & 0.13 & 0.09 & "146.74 & 8.77 & 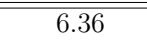 & 137.94 & 137.98 & 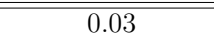 & 0.01 \\
\hline 175.72 & 175.51 & 0.21 & 0.12 & 185.47 & 9.75 & 5.55 & 175.64 & 175.74 & 0.05 & 0.01 \\
\hline 136.95 & $: 136.80$ & 0.15 & 0.11 & 145.74 & 8.79 & 6.42 & $: 136.86$ & 136.96 & 0.08 & 0.01 \\
\hline 187.44 & 186.78 & 0.66 & 0.35 & 196.34 & 8.90 & 4.75 & $\begin{array}{l}187.26 \\
\end{array}$ & 187.51 & 0.13 & 0.04 \\
\hline 162.33 & 161.61 & 0.72 & 0.44 & 174.28 & 11.95 & 7.36 & 162.19 & 162.33 & 0.09 & 0.00 \\
\hline 166.24 & $\begin{array}{l}165.97 \\
\end{array}$ & 0.27 & 0.16 & 176.69 & 10.45 & 6.29 & 166.12 & 166.25 & 0.08 & 0.01 \\
\hline avg & ! & & 0.21 & & & 6.12 & । & & 0.08 & 0.01 \\
\hline
\end{tabular}

horizon approach.

For the instances tested in Table 9, EVPI value is small, implying that the solution of our stochastic model is close to the one obtained with perfect information.

\section{Conclusion}

In this study, we presented a model for demand response optimization in which we considered all types of appliances already mentioned in the literature as well as renewable energy and batteries. We also incorporated the uncertainty in temperature and the availability of renewable energy using multi-stage stochastic programming. We provided a decomposition scheme that produced good quality bounds for large instances. We also conducted a detailed experiment that provided insights about the gains in cost and utility for residents with different price sensitivity. We observed that optimization can provide significant improvements both in cost and utility for all types of residents. The same is also true for renewable energy and batteries. With these three tools combined, the peak demand can be reduced significantly, providing savings for residents, flexibility for the electricity providers and less damage to the environment.

One of the major limitations of our study is that we consider only the power flow from utilities to customers. Investigating the impact of bidirectional power flow is an important future research direction. It may be interesting to compare the amount of energy sent to the grid by residents with different price sensitivities in different pricing schemes.

Another promising direction of future work is to investigate the integration of the electric vehicles in the power grid. Although they can be modelled in the same way 
as appliances with battery, considering the uncertainty in the availability of vehicle may be interesting.

From the computational point of view, as a future work, parallel computing may be exploited to compute lower and upper bounds to shorten the required computational time.

\section{Acknowledgement}

Part of the research of the first and third authors was done at the Department of Industrial Engineering, Bilkent University.

\section{References}

[1] J. Conti, P. Holtberg, J. Diefenderfer, A. LaRose, J. T. Turnure, L. Westfall, International energy outlook 2016 with projections to 2040, Tech. rep., USDOE Energy Information Administration (EIA), Washington, DC (United States). Office of Energy Analysis (2016).

[2] U. EIA, Electric power sales, revenue, and energy efficiency form eia-861 detailed data files (2017).

[3] A.-H. Mohsenian-Rad, V. W. Wong, J. Jatskevich, R. Schober, Optimal and autonomous incentive-based energy consumption scheduling algorithm for smart grid, in: Innovative Smart Grid Technologies (ISGT), 2010, IEEE, 2010, pp. 1-6.

[4] A.-H. Mohsenian-Rad, V. W. Wong, J. Jatskevich, R. Schober, A. Leon-Garcia, Autonomous demand-side management based on game-theoretic energy consumption scheduling for the future smart grid, IEEE transactions on Smart Grid 1 (3) (2010) 320-331.

[5] P. Samadi, A.-H. Mohsenian-Rad, R. Schober, V. W. Wong, J. Jatskevich, Optimal real-time pricing algorithm based on utility maximization for smart grid, in: Smart Grid Communications (SmartGridComm), 2010 First IEEE International Conference on, IEEE, 2010, pp. 415-420.

[6] N. Gatsis, G. B. Giannakis, Residential demand response with interruptible tasks: Duality and algorithms, in: Decision and Control and European Control Conference (CDC-ECC), 2011 50th IEEE Conference on, IEEE, 2011, pp. 1-6. 
[7] X. Chen, T. Wei, S. Hu, Uncertainty-aware household appliance scheduling considering dynamic electricity pricing in smart home, IEEE Transactions on Smart Grid 4 (2) (2013) 932-941.

[8] H. Goudarzi, S. Hatami, M. Pedram, Demand-side load scheduling incentivized by dynamic energy prices, in: Smart Grid Communications (SmartGridComm), 2011 IEEE International Conference on, IEEE, 2011, pp. 351-356.

[9] Z. Zhao, W. C. Lee, Y. Shin, K.-B. Song, An optimal power scheduling method for demand response in home energy management system, IEEE Transactions on Smart Grid 4 (3) (2013) 1391-1400.

[10] A.-H. Mohsenian-Rad, A. Leon-Gracia, Optimal residential load control with price prediction in real-time electricity pricing environments, IEEE Transactions on Smart Grid 1 (2010) 120 - 133.

[11] K. Ma, T. Yao, J. Yang, X. Guan, Residential power scheduling for demand response in smart grid, International Journal of Electrical Power \& Energy Systems 78 (2016) 320-325.

[12] R. Energy, Energy efficiency trends in residential and commercial buildings.

[13] S. Althaher, P. Mancarella, J. Mutale, Automated demand response from home energy management system under dynamic pricing and power and comfort constraints, IEEE Transactions on Smart Grid 6 (4) (2015) 1874-1883.

[14] H. T. Nguyen, D. T. Nguyen, L. B. Le, Energy management for households with solar assisted thermal load considering renewable energy and price uncertainty, IEEE Transactions on Smart Grid 6 (1) (2015) 301-314.

[15] S. Tiptipakorn, W.-J. Lee, A residential consumer-centered load control strategy in real-time electricity pricing environment, in: Power Symposium, 2007. NAPS'07. 39th North American, IEEE, 2007, pp. 505-510.

[16] N. Li, L. Chen, S. H. Low, Optimal demand response based on utility maximization in power networks, in: Power and Energy Society General Meeting, 2011 IEEE, IEEE, 2011, pp. 1-8.

[17] K. M. Tsui, S.-C. Chan, Demand response optimization for smart home scheduling under real-time pricing, IEEE Transactions on Smart Grid 3 (4) (2012) $1812-1821$. 
[18] M. Shafie-Khah, P. Siano, A stochastic home energy management system considering satisfaction cost and response fatigue, IEEE Transactions on Industrial Informatics 14 (2) (2018) 629-638.

[19] F. Hafiz, A. R. de Queiroz, I. Husain, Multi-stage stochastic optimization for a pv-storage hybrid unit in a household, in: 2017 IEEE Industry Applications Society Annual Meeting, IEEE, 2017, pp. 1-7.

[20] F. Hafiz, A. R. de Queiroz, P. Fajri, I. Husain, Energy management and optimal storage sizing for a shared community: A multi-stage stochastic programming approach, Applied energy 236 (2019) 42-54.

[21] A. Bhattacharya, J. P. Kharoufeh, B. Zeng, Managing energy storage in microgrids: A multistage stochastic programming approach, IEEE Transactions on Smart Grid 9 (1) (2016) 483-496.

[22] P. Aliasghari, B. Mohammadi-Ivatloo, M. Alipour, M. Abapour, K. Zare, Optimal scheduling of plug-in electric vehicles and renewable micro-grid in energy and reserve markets considering demand response program, Journal of Cleaner Production 186 (2018) 293-303.

[23] H. Allcott, Real time pricing and electricity markets, Harvard University 7.

[24] S. Borenstein, Equity effects of increasing-block electricity pricing.

[25] A. Illinois, Real-time pricing for residential customers, http://www.ameren. com/Residential/ADC_RTP_Res.asp (2009).

[26] B. Hydro, BC Hydro Annual Report 2009, BC Hydro, 2009.

[27] B. Sandikçi, O. Y. Özaltin, A scalable bounding method for multistage stochastic programs, SIAM Journal on Optimization 27 (3) (2017) 1772-1800.

[28] F. Maggioni, E. Allevi, M. Bertocchi, Monotonic bounds in multistage mixedinteger stochastic programming, Computational Management Science 13 (3) (2016) 423-457.

[29] F. Maggioni, G. C. Pflug, Bounds and approximations for multistage stochastic programs, SIAM Journal on Optimization 26 (1) (2016) 831-855.

[30] M. J. Reno, C. W. Hansen, J. S. Stein, Global horizontal irradiance clear sky models: Implementation and analysis, SANDIA report SAND2012-2389. 
[31] NREL, Nsrdb data viewer, retrieved February 8, 2018, https://maps.nrel. gov/nsrdb-viewer (2018).

[32] R. D. Cess, M. Zhang, P. Minnis, L. Corsetti, E. Dutton, B. Forgan, D. Garber, W. Gates, J. Hack, E. Harrison, et al., Absorption of solar radiation by clouds: Observations versus models, Science 267 (5197) (1995) 496-499.

[33] S.-C. Tsay, M. D. King, R. F. Cahalan, W. K.-M. Lau, Absorption of solar radiation by clouds: A second look at irradiance measurements.

[34] L. F. Escudero, A. Garín, M. Merino, G. Pérez, The value of the stochastic solution in multistage problems, Top 15 (1) (2007) 48-64. 